\title{
Preface: special issue on geo-social media analytics
}

\author{
Feng Chen ${ }^{1}$ • Arnold Boedihardjo ${ }^{2}$ - Chang-Tien Lu $^{3}$
}

Published online: 27 June 2018

(C) Springer Science+Business Media, LLC, part of Springer Nature 2018

Advancements in communication infrastructures and low access barriers to communication sinks (e.g., personal mobile devices) have dramatically increased the size and reach of open source data such as those observed in social media: Twitter feeds, user blogs, Flikr images, and others. In several cases, the data have been implicitly or explicitly encoded with spatial and temporal attributes manifested in a variety of forms such as place names in Tweets and GPS coordinates in Flikr. Exploiting the open source data in conjunction with their spatiotemporal contexts can enhance our understanding of the physical environment, societal condition, and the dynamic and complex relationships between them. For example, in the context of disaster response, Twitter feeds and Flikr imageries can provide a rich and valuable avenue for monitoring the spatial distribution of affected areas and population sentiments to positively impact relief efforts such as the one caused by Hurricane Sandy. Also, during Arab Spring, the geographic evolution of population attitudes as observed in various social mediums can provide effective indicators of demonstrations and protests. These examples underline the importance of geo-social media in bringing awareness, insights, and decisions to impact these major events.

This special issue includes five articles delving on the following themes of geo-social media data: understanding complex patterns and relationships between humans and their environment,

Guest Editors: Feng Chen, Arnold Boedihardjo, Chang-Tien Lu

Chang-Tien Lu

ctlu@vt.edu

Feng Chen

fchen5@albany.edu

Arnold Boedihardjo

arnold.boedihardjo@radiantsolutions.com

1 Department of Computer Science, University at Albany - SUNY, 1215 Western Ave, Albany, NY 12222, USA

2 Radiant Solutions, 2325 Dulles Corner Blvd, Herndon, VA 20171, USA

3 Department of Computer Science, Virginia Tech, Northern Virginia Center, 7054 Haycock Road, Falls Church, VA 22043, USA 
addressing the challenges of highly skewed data availability and distributions, optimizing analytical models to handle continuously evolving data, inferring and extracting implicit and ambiguous spatiotemporal attributions, and providing robust analytics in noisy data.

The paper by Guo et al. ("Human Mobility Semantics Analysis: a Probabilistic and Scalable Approach") leverages full Bayesian sequential modeling to discover cascading movement patterns in highly noisy mobile data. Another important form of geo-social data is location-based social network (LBSN). The paper by Li et al. ("Assessing spatiotemporal predictability of LBSN: A case study of three Foursquare datasets") performs data analysis to understand and quantify the spatiotemporal predictability of LBSN. Extracting and estimating the underlying correlation within the data allows for a realistic assessment on the quality of predictions that can be made from applicable techniques.

Geocoding in social media data has attracted tremendous attention due to the improved analysis that can be brought to be bear with accurate spatial contextualization. The paper entitled "Strategies for Combining Twitter user geo-location methods" by Ribeiro and Pappa evaluates and compares sixteen existing methods for user location inference based on different information sources, and develops four methods to combine the outputs of the evaluated methods to improve accuracy and recall. A related topic, in the paper entitled "A Framework for Annotating OpenStreetMap Objects Using Geo-tagged Tweets" by Chen et al., proposes a comprehensive framework to integrate social media with OpenStreetMap (OSM) to derive knowledge about geographical objects, specifically, top relevant annotations from tweets for objects in OSM.

Lastly, focusing on developing fundamental computational methods to support automatic analyses is the paper by Kanza and Shalem ("Combined Geo-social Search: Computing Top-k Join Queries over Incomplete Information"). This paper studies how search queries based on spatial and social attributes can be performed efficiently and effectively in geo-social datasets that associate people and activities with locations.

We hope that these papers will inspire the scientific community to further conduct research in the area of geo-social media analytics. We are grateful to the authors and referees for their tremendous contributions and efforts towards making this special issue possible.

\section{Publisher's Note}

Springer Nature remains neutral with regard to jurisdictional claims in published maps and institutional affiliations. 


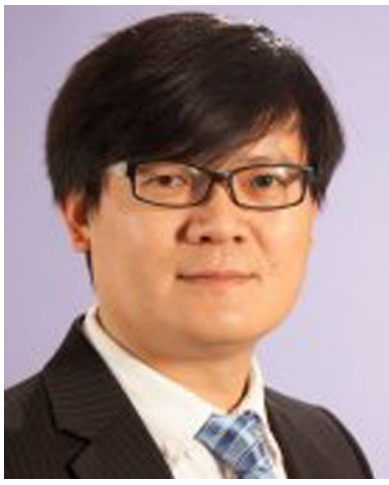

Feng Chen is an Assistant Professor of Computer Science at the University of New York at Albany - SUNY, Albany, NY, USA. He received his Ph.D. degree in computer science from Virginia Tech, Blacksburg, VA, USA, in 2012. He is the recipient of the NSF CAREER award in 2018. His research interests include spatial databases, anomalous pattern detection, event detection and forecasting, and graph mining. His research has been supported by NSF, NIH, ARO, IARPA, and the U.S. Department of Transportation.

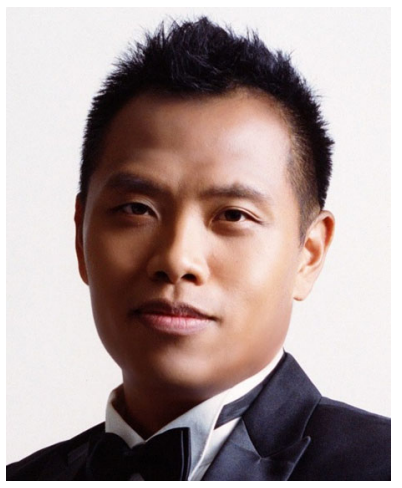

Arnold Boedihardjo received his $\mathrm{PhD}$ degree in Computer Science from Virginia Tech in 2010. He is currently a principal R\&D scientist at Radiant Solutions. Prior to joining Radiant Solutions, he was a research scientist at the US Army Engineer and Research Development Center. His research interests include machine learning, geospatial data mining, big data analysis, and information retrieval. 


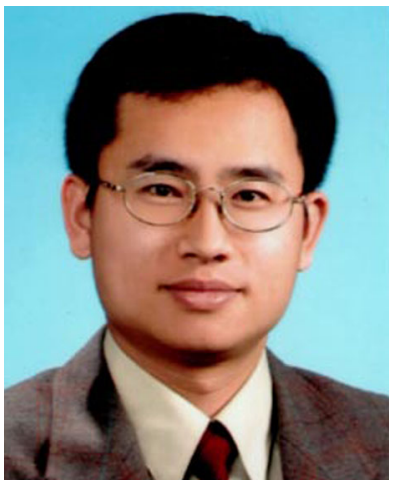

Chang-Tien Lu is a Professor of Computer Science and Associate Director of the Discovery Analytics Center at Virginia Tech. He received his Ph.D. from the University of Minnesota at Twin Cities in 2001. He served as Program Chair of the 18th IEEE International Conference on Tools with Artificial Intelligence in 2006, and General Chair of the 17th ACM SIGSPATIAL International Conference on Advances in Geographic Information Systems in 2009 and the International Symposium on Spatial and Temporal Databases in 2017. He also served as Secretary (2008-2011) and Vice Chair (2011-2014) of the ACM Special Interest Group on Spatial Information (ACM SIGSPATIAL). His research interests include spatial databases, data mining, urban computing, and intelligent transportation systems. He is an ACM Distinguished Scientist. 